\title{
Necessity or Vanity: Designer Babies, CRISPR, and the Future of Genetic Modifications
}

\author{
Fawzaan M. Hashmi \\ Mankato West High School, Mankato, Minnesota 56001, USA
}

\begin{abstract}
CRISPR technology can drastically speed up the pace of genetic experimentation and could possibly eliminate many hereditary diseases from bloodlines. CRISPR was discovered only by chance in microbes; experimenters eventually managed to apply the technique to mammalian genes. However, CRISPR is still very much underdeveloped, as in many areas it lacks in precision and efficiency. For humans, the main application of CRISPR is to eliminate hereditary disease, but it has also stimulated the urge for the bête noire of creating genetically modified children, called designer babies. The idea of designer babies is controversial, as to some, the genetic modification of humans is ethically very troubling. The majority of scientists believe that there needs to be a moratorium on the generation of genetically modified babies, but there are a select few with the exact opposite view. Majority of scientists insist that we cannot stop the advancement of contemporary technology and must go full force ahead. This paper attempts to trace the emergence of CRISPR and its potential utility for addressing human disease alongside its deficiencies and the ethical problems of designer babies. Lastly, recommendations are made to expedite CRISPR related future research and utilizing the technology for the benefit of generations to come.
\end{abstract}

Key Words: CRISPR, designer babies, genetic modifications, hereditary diseases, Cas9, eugenics, germline modification

\section{Introduction}

Imagine a world where parents can design babies who are intelligent, good-looking, and free from any hereditary disease. These desires might be realized if the scientific advancements in genetic editing technology continue at an accelerated pace and our civilization resolves the ethical dilemma associated with the potential abuse of this technology. CRISPR (Clustered Regularly Interspaced Short Palindromic Repeats) is a gene-editing technology that utilizes the Cas9 protein (it should be noted that since the making of this paper, a new, more efficient and precise protein called Cas12a has been discovered) ("CRISPRCas12a More Precise Than CRISPR-Cas9"). CRISPR works by using three main tools: A GPS for locating DNA, scissors for cutting DNA (deleting segments), and a pen for writing new DNA (inserting segments) (Wright et al. 2016). A repair template tailored to specific parts of a DNA sequence guides Cas9. CRISPR sequences are made into short RNA (ribonucleic acid) sequences in order to match the DNA sequences. After an action similar to a GPS, the DNA is found. Then, Cas9 connects to and cuts the DNA, which shuts down the gene. Researchers can test the genome's function by using modified versions of the protein Cas9 that do not run the risk of actually cutting through the DNA (Wright et al. 2016).

\section{History}

CRISPR has a rich and surprisingly long history. Researchers in Osaka, Japan, in 1987 accidentally recorded DNA clusters, and, independently, similar clusters were found in the historic port city of Alicante, Spain, in 1993 (Zimmer 2015, Lander 2016). Researchers here unearthed microbes that safeguarded themselves with an adaptive immune system that scoured the DNA sequences of comminatory viruses. Amazingly, CRISPR changed from this into a way of editing living cells in a span of just three years. The manner in which 
researchers formulated CRISPR reflected the swelling importance in the biology of big data. The discovery of biological processes important to CRISPR came from large and public accumulations of biological data, not conventional lab experiments (Lander 2016).

\section{Development of CRISPR and Designer Babies}

Genetic modification involves the rearrangement of nucleotides in order to construct new DNA; the first of these recombinant DNA molecules were synthesized in 1972 and the first genetically modified organism (GMO) was a 1973 bacterium (Jackson et al. 1972, Arnold 2009). Since then, GMOs have controversially been applied to plants and animals in order to increase their yield and/or the tolerance of products for human consumption. GMOs have faced varying levels of success and acceptance; GMO foods are stringently regulated in the European Union, for instance (Davison 2009). Genetic modifications on animals can also help in eradicating human diseases by analyzing human genes and methodically activating and disabling targeted genes. Further medical applications of genetic engineering include the genetic enhancement of proteins and drugs as well as somatic gene therapy (the use of genetic material to treat human disease). However, scientists could not effectively use CRISPR, a form of genetic modification for editing genomes (potentially inheritably) until almost twenty years after it was discovered (Lander 2016).

Scientists tried to apply the techniques of CRISPR initially used on microbes to mammals and, by extension, to humans. Scientists in the late 1980s discovered a way of editing mammalian genomes in living cells. This was a miracle for its time; however, this process was extremely inefficient because the frequency of homologous recombination, where natural genes were switched with modified variants, was low. A "meganuclease" was found to increase the frequency of homologous recombination by establishing doublestranded breaks (Lander 2016). In order to get an efficient method for editing mammalian genomes, scientists needed a no-fail method to produce a double-stranded break wherever they wanted. The first strategy was to use zinc-finger nucleases, which are binding proteins. Despite being promising, getting these zinc-finger nucleases to consistently recognize the necessary genomic loci was an arduous process. Translating CRISPR's method of cutting and editing genomic loci to mammalian cells was difficult; their genomes are a thousand times larger and reflect a more complicated chromatin structure (Lander 2016). Rudimentary microbe systems could not be serviceably applied to this au courant environment.

Increasing the number of Cas9 proteins that would enter into the nucleus was fundamental to improving CRISPR's yield in mammal cells. In 2012, researchers developed a functioning three-component system of Cas9, incorporating S. pyogenes variants (S. thermophilus Cas9 proteins concentrate in nucleoli, while S. pyogenes Cas9 proteins are more evenly spread and thereby better suited for mammalian biologies). From this moment on, genes could be modified productively with deletions, using non-homologous end-joining, and insertions, catenating sequences with guided homologous recombination. Excitingly, genes could be compiled concurrently because of spacers embedded in CRISPR arrays (Lander 2016). CRISPR could now be used to concoct complex mouse models of inherited diseases and to implement genome-wide screening.

\section{Clinical Benefits of CRISPR}

More and more people are becoming familiar with CRISPR, and that number is only growing. CRISPR is a substantially more efficient way to modify the human genome than previous technologies. Due to CRISPR, genetic experiments that took years can now take only a few weeks. Many hereditary diseases such as lung cancer, liver disease, congenital blindness, sickle cell disease, and Huntington's disease can be eliminated by CRISPR (Wright et al. 2016). As an added bonus, CRISPR can permanently remove the disease from the bloodline, so these debilitating diseases would not affect those future generations.

CRISPR can also be used to help diseases like ALS and types of muscular dystrophy. These are caused by RNA build-ups and proteins being somewhere where they do not belong. CRISPR can help eradicate the damaging effects of the RNA, thus reversing the devastating effects. The goal is to nullify such diseases but CRISPR cannot target the DNA; it has to target the RNA, which is a messenger molecule. However, it is not very easy to simply cut off RNA when it is conjoined with DNA. Therefore, a different Cas9 protein must be applied to separate DNA and RNA (Mullin 2017). After the separation of DNA and RNA has been achieved, CRISPR can bind to and delete the desired RNA. 


\section{Impediments to Clinical Applications}

Many of the present-day debates around CRISPR rehash those of gene therapy. Excessive expectations for gene therapy led to an irrevocable letdown for a supportive public. Impediments to beneficial gene therapy included the eruption of childhood leukemia when the viral vector for gene therapy inadvertently triggered a dormant oncogene (a gene that can cause cancer) (Mulvihill et al. 2017). These problems should serve as a caution for today's advocates of CRISPR.

It is, after all, difficult for clinical applications of CRISPR to reach a desirable level of precision. CRISPR can precisely target almost any nucleotide sequence of short length (this includes entire chromosomes). Many frequently occurring disorders rely on these nucleotide sequences. The mutations of introns (once thought to be "junk DNA") and a whole host of disparate segments of genetic processes have been shown to be pathogenic (Wright et al. 2016). Clinicians also need to take pseudogenes, gene fragments that are evolutionarily prior to the functional genes they resemble and are often still present, into account. In fact, complex and opaque relations between genes and the environment precipitate most diseases. Discerning which sequences lead to disease is, therefore, an onerous task.

Although Cas9 has a plethora of uses for CRISPR research, it is still not the most practical in a clinical setting. With Cas9, it has proven difficult to repair DNA sequences with the preferred degree of homology across the ends of DNA segments. Developing forms of Cas9 that are sensitive to light and chemical substances might better CRISPR's potential and address its failings in clinical precision (Wright et al. 2016).

Transporting Cas9 is another major obstacle. Researchers have in turn tried to make use of smaller Cas9 orthologs, variations that share Cas9's function, for easier transport. In addition to Cas9, the Cas6f/Csy4, Cascade, and Cpf1 genes can be explored in order to improve CRISPR systems (Wright et al. 2016). Additionally, the regulation of CRISPR-Cas systems is very much unstudied. And yet, the research that has taken place does seem to suggest that the regulatory methods of CRISPR-Cas systems can be more efficient and dynamic than expected.

CRISPR-Cas systems, made up of CRISPR arrays and Cas genes, should be expected to defend against foreign and unwanted DNA. Spacers throughout the CRISPR array correspond to specific segments of invading DNA so that the system is immune to infection from those foreign segments. Cas proteins can help adapt the system to new invading genomes by adding new spacers to this array. Because of large existing collections of experimental data for CRISPR transcription, researchers should be able to model CRISPR-Cas regulation, and the existing evidence demonstrates that this regulation essentially involves an on/off mechanism. And because small increases in the rate of CRISPR transcription significantly speed up the transcription of pre-CRISPR RNA, of which a certain amount is needed in order to turn the switch "on," this system is effective in developing the protecting spacers (Blagojevic et al. 2017). Although it is difficult at this stage to precisely map the signaling sequence of CRISPR-Cas regulation, mathematical modeling can nevertheless isolate the efficiency of this binary on/off regulation. Despite all the biological and clinical impediments that justify further investigation into the application of CRISPR technology, the straightforward ability of CRISPR-Cas systems to protect themselves against contaminating DNA is another reminder of why this technology might very well be worth all the trouble.

\section{Ethical Considerations}

CRISPR has by now taken the scientific community by storm. However, whenever modifying the human germline is brought up, contentious international debates ensue. Many people do not support the modification of the human race. They argue that there are always unintended consequences for every change imprinted onto the human genome (Knoepfler 2017). And in fact, prior to that stage, the modification of the embryo itself fails about half the time (Mulvihill et al. 2017).

The editing of genomes for plants and animals has been around for a long time, but it can now be applied to humans. But how far we should take this technique is subject to a host of ethical conflicts. Everybody from theologians to doctors has been involved in a debate over the ethical and social consequences of human 
genetic modification as well as its relationship with eugenics. Germany and Denmark have banned the creation of genetically modified babies, largely due to the belief that modifying an embryo would ruin the already healthy embryo (Knoepfler 1-10). Genetically editing an already alive human may be deemed to be even scarier as we would have no idea what the consequences and repercussions might be. These debates of medical ethics cannot be addressed fully without introducing the Eugenics movement and the debate around imposing a moratorium on future CRISPR research.

\section{A- Eugenics}

The American eugenics movement of the last century concentrated on "purifying" bloodlines from the "unhealthy" and sometimes straightforwardly "undesirable" characteristics of non-white, disabled, and poor populations. Support for eugenics in American culture was widespread in the early part of the 20th century. People competed to discover who had healthier children with "Better Baby Contests," in which children were evaluated alongside vegetables (Bouche and Rivard 2014).

Twenty-eight (28) states (Indiana being the first) by 1931 passed sterilization laws so that 64,000 were forcibly sterilized on American soil. In Buck v. Bell (1927), the state of Virginia tried to sterilize Carrie Buck. Supreme Court Justice Wendell Holmes sided with Virginia, legalizing all the new sterilization laws, and wrote, "three generations of imbeciles is enough (Bouche and Rivard 2014)." The cruelty of California's forced sterilization inspired the Nazis' eugenics programs. Beginning in the 1940s and especially with the recognition of the severity of Nazi programs, eugenics fell out of favor.

It is argued that if genetically modified designer babies are permitted to all interested parents it might serve as a backdoor to modern eugenics. A designer baby is a genetically modified human embryo, usually created in an artificial womb. A designer baby can have whatever traits the parent or scientist wants, making it possible to have a "superhuman" (Knoepfler 1-10). For instance, parents could make a baby tall, smart, athletic, handsome, etc. After all, parents would conceivably want the best for their kids, which would entail wanting their kids to be at the top.

Proponents of CRISPR technology argue that the process is inefficient and would take a huge sum of money at the beginning to create a designer baby, despite saving a consequential amount on healthcare costs since in theory the child's germline could be edited to make them mostly immune to common viruses as well as serious diseases. Opponents still feel that CRISPR technology can be manipulated by the wealthy few to create a perfect human being, possibly capable of dominating billions of impoverished people. In the worstcase scenario, some would use gene editing to try to eliminate everyone in the world who is not a designer baby in order to try to create a sort of "perfect race."

\section{B- Need for Moratorium}

Germline genomes mutate, and there are many likely associations of spontaneous genetic disease with environmental factors (Mulvihill et al. 2017). This paradox exemplifies an uncertainty regarding how germline genomes work. For many specialists, in fact, the uncertainty of outcomes justifies a moratorium on the clinical use of CRISPR. Proponents of a moratorium believe that several factors such as Federal Drug Agency (FDA) policies, civil liability, possible criminal charges, professional sanctions, and institutional pressures already buttress such a moratorium (Knoepfler 97-8).

In 2017, the National Academy of Sciences (NAS) published a report supporting the testing of CRISPR gene editing for serious hereditary diseases such as sickle cell disease, hemophilia, cystic fibrosis, Duchenne muscular dystrophy, genetic forms of blindness, and cancer, and not for "stronger" or "smarter" designer babies (Powledge 2017). However, according to writer and researcher Paul Knoepfler, who has called for a moratorium on all germline modification period, the report assigns too much importance to the specter of parental autonomy and does not appreciate the level of testing required before embryo editing could medically be considered (Knoepfler 2017). The NAS report recommendations, like the one proposed in 2014 by a group of biologists led by Professor Jennifer Doudna, would be applied to any possible clinical usage on CRISPR-style gene editing and has allowed the current pace of testing on mammalian, including human, cells to continue (Doudna 2017). 
Doudna and Sternberg (2017) struggled on the debate between a moratorium and increased research for CRISPR technology. Both of the authors are pioneers in CRISPR research in the recent past and have lately advocated for more restrictions on future research. Doudna and Sternberg (2017) have summarized their dilemma by defining CRISPR as something that "offers both the greatest promise and, arguably, the greatest peril for the future of humanity (Doudna and Sternberg 2017)." In January 2015, eighteen scientists including Professor Doudna recommended that CRISPR-related ethical issues must be thoroughly investigated and understood before any attempts at human engineering are sanctioned. These scientists suggested restrictions but fell short of arguing for complete restrictions on future research.

\section{Recommendations and Future Directions}

CRISPR has elevated hopes of permanently protecting humans from genetic disease. Yet, so much is unknown about the relations between CRISPR methods and environmental factors as well as between the internal variations of the genetic code. Because this technology has a potential to be utilized for a variety of harmful and problematic manipulations of human populations, governments and institutions should take the proactive approach and direct scientists to address only hereditary diseases to reduce sufferings and not just for the sake of vanity. A harrowing example of the latter is the case of He Jiankui, who made what were allegedly the first CRISPR edits of humans by irresponsibly and gratuitously deactivating the genes of a pair of twins (Yong 2018).

After careful review of the current state of the CRISPR related scientific progress and the potential to benefit the human race, this study makes the following recommendations to implement CRISPR in an influential and ethical way.

- Investment: A good start for universities, national governments, and global institutions is to invest more in furthering our knowledge of CRISPR. All new technologies of the last century needed public-sector support to be fully developed. The initial financial cost often outweighs potential benefits in the years to come.

- Education: National governments should educate citizens, the insurance industry, and patients about the cost, risks, ethical considerations and possibilities of CRISPR technology. Universal awareness of this revolutionary technology reduce fear on the part of informed citizens and help them keep an eye on the abusers. Another related solution is to educate high school and college students along with science teachers about the possibilities of this technology and its career paths. This way, the next generation of people will be able to make informed decisions in their adulthood, considering that CRISPR might be more prevalent by the time the current generation of children are adults. In addition, medical institutions, universities, and insurance providers need to teach their concerned clients about the possibilities of CRISPR technology, especially if they end up considering it for clinical purposes.

- Regulations: National governments and global institutions should set ethical guidelines for research institutions and universities. Although it would be difficult to enforce these ethical guidelines outside domestic regulatory regimes, scientists across the world did remain faithful to a 1975 moratorium on the recombinant DNA technique (Wade 2015). Likewise, because the debates surrounding CRISPR deal with the fundamentals of the human genetic makeup, the entire world has a significant stake in how this technology evolves. The precedent suggests that the majority of credentialed researchers would have faith in consensus. At the same time, something more taxing, such as a multinational agreement, might need to be organized in order to reel in rogue experimenters around the world. Moreover, any ethical framework must aggressively ensure that no discriminatory beliefs influence the direction of modern technology. Using CRISPR for any racist or sexist purposes would be a backdoor to eugenics.

- Coordination: The true benefits of CRISPR technology can only be drawn through close coordination among geneticists, biologists, medical ethics experts, religious thinkers, economists, educationalists, and policymakers in all technologically advanced countries. Without a close working relationship, there is a serious risk of misinformation and eventual delays in enjoying the full potential of this technology. This study suggests the creation of a high-power commission in the United States and other interested 
countries where representatives from various disciplines can discuss controversial issues and chart out a strategic plan for the next decade. The policy recommendations and practical steps can be revised on a periodic basis to accommodate the changing technological and ethical landscape.

- Cost \& Benefit Analysis: National governments and researchers should analyze and compare the initial investment to develop CRISPR technology and the potential savings not only in healthcare costs but also from making millions of citizens productive again. Without a detailed cost-benefit analysis, it may be difficult to secure major findings in today's competitive economic environment.

Scientific researchers, the insurance industry, medical institutions, institutions of higher education, policymakers around the world, and global institutions can use the findings and recommendations of this study for the future development of CRISPR technology and the benefit of their clients. There are obviously challenges ahead, but the possibilities outweigh any obstacles and economic costs.

Throughout human history, new technologies tended to be used for the benefit of humanity in general. Unfortunately, the same technology has also been employed for destructive purposes by iniquitous people in power. CRISPR technology is no different from other almost revolutionary technologies of the past including nuclear technology. Therefore, scientists must learn from their experiences and use CRISPR technology to improve the quality of life of millions of people by avoiding debilitating diseases. We must not see the CRISPR technology just as a vanity project to improve the physical attributes of a select few but rather as a necessity for our time.

\section{References}

1. Arnold, Paul. "Timeline of Genetic Engineering." Bright Hub, 12 Nov. 2009, www.brighthub.com/science/genetics/articles/21983.aspx.

2. Bouche, Teryn, and Laura Rivard. "America's Hidden History: The Eugenics Movement." Nature News, Nature Publishing Group, 18 Sept. 2014, www.nature.com/scitable/forums/genetics-generation/americas-hidden-history-the-eugenics-movement-123919444.

3. "CRISPR-Cas12a More Precise Than CRISPR-Cas9." GEN, GEN - Genetic Engineering and Biotechnology News, 20 Nov. 2018, www.genengnews.com/topics/genome-editing/crispr-cas12a-moreprecise-than-crispr-cas $9 /$.

4. Davison, John. "GM Plants: Science, Politics and EC Regulations." Plant Science, Elsevier, 24 Dec. 2009, www.sciencedirect.com/science/article/pii/S0168945209003112.

5. Doudna, Jennifer, and Samuel Sternberg. A Crack in Creation: A New Power to Control Evolution, Vintage Publishing, 2017.

6. Jackson, David A., et al. "Biochemical Method for Inserting New Genetic Information into DNA of Simian Virus 40: Circular SV40 DNA Molecules Containing Lambda Phage Genes and the Galactose Operon of Escherichia Coli." Proceedings of the National Academy of Sciences of the United States of America, U.S. National Library of Medicine, Oct. 1972,

www.ncbi.nlm.nih.gov/pmc/articles/PMC389671/.

7. Knoepfler, Paul. GMO Sapiens: the Life-Changing Science of Designer Babies. World Scientific, 2016.

8. Knoepfler, Paul. "National Academies Panel Leaves Door a Crack Open to Future Human Genetic Modification." The Niche, 14 Feb. 2017, ipscell.com/2017/02/national-academies-panel-leaves-door-acrack-open-to-future-human-genetic-modification/.

9. Lander, Eric S. "The Heroes of CRISPR.” Cell, Cell Press, 14 Jan. 2016, www.sciencedirect.com/science/article/pii/S0092867415017055.

10. Mullin, Emily. "A Startup Wants to Embed CRISPR in the Body to Fight Huntington's and ALS." MIT Technology Review, MIT Technology Review, 6 Oct. 2017, www.technologyreview.com/s/608967/arming-bodies-with-crispr-to-fight-huntingtons-disease-and-als/

11. Mulvihill, John J., et al. "Ethical Issues of CRISPR Technology and Gene Editing through the Lens of Solidarity | British Medical Bulletin | Oxford Academic." OUP Academic, Oxford University Press, 23 Feb. 2017, academic.oup.com/bmb/article/122/1/17/3045812. 
12. Powledge, Tabitha M. "Designer Babies OK' CRISPR Patent Award.” On Science Blogs, 18 Feb. 2017, blogs.plos.org/onscienceblogs/2017/02/17/genome-editing-of-designer-babies-ok-in-some-cases-hugecrispr-patent-fight-decision-but-whos-the-ultimate-winner/.

13. Wade, Nicholas. "Scientists Seek Ban on Method of Editing the Human Genome." The New York Times, The New York Times, 19 Mar. 2015, www.nytimes.com/2015/03/20/science/biologists-call-for-halt-togene-editing-technique-in-humans.html?_r=0

14. Wright, Addison V., et al. "Biology and Applications of CRISPR Systems: Harnessing Nature's Toolbox for Genome Engineering." Cell, Cell Press, 14 Jan. 2016, www.sciencedirect.com/science/article/pii/S0092867415016992.

15. Yong, Ed. "The CRISPR Baby Scandal Gets Worse by the Day." The Atlantic, Atlantic Media Company, 5 Dec. 2018, www.theatlantic.com/science/archive/2018/12/15-worrying-things-about-crisprbabies-scandal/577234/.

16. Zimmer, Carl. "CRISPR Natural History in Bacteria.” Quanta Magazine, 6 Feb. 2015, www.quantamagazine.org/crispr-natural-history-in-bacteria-20150206/. 